\title{
On asymptotic development for solutions of ordinary differential equations
}

\author{
by CIN HUa Szu (Peking)
}

In recent years there have been papers dealing with problem of asymptotic development for solutions of ordinary differential equations. This problem was considered by Kamke [4], Bellman [1] and Levinson [3].

For the equation $|x|^{r} y^{\prime}=f(x, y)$, where the right-hand member is a polynomial in $y$ with coefficients possessing an asymptotic development as $x / 0$, Bruijn [2] has shown the existence of a solution $y(x)$ possessing an asymptotic development as $x \nearrow 0$.

The present paper concerns a more general case; namely, we consider a system of ordinary differential equations whose right-hand members are not necessarily polynomials but possess an asymptotic development into a series of several variables.

We shall give a sufficient condition for systems of ordinary differential equations to possess integrals having an asymptotic development.

We shall compute the coefficients of these asymptotic developments by the method of undetermined coefficients. Changing variables we transform this problem into an existence problem for integrals possessing an asymptotic development with null cocfficients. In the proof of existence for these integrals T. Ważewski's topological method is applied.

Under certain additional conditions we characterize the set formed by integrals tending to the origin and possessing an asymptotic development at $x=0$.

\section{\$1. Notation and definitions.}

1. We introduce the following notation:

$y=\left(y_{1}, \ldots, y_{n}\right), \quad Y=\left(y_{0}, y\right), \quad y \in R^{n}, \quad Y \in R^{n+1} ; \quad|Y|=\left(\sum_{i=0}^{n} y_{i}^{2}\right)^{1 / 2}$.

By $O_{n}, O_{n+1}$ we denote the origins of $R^{n}, R^{n+1}$ respectively.

We shall use $n \times n$ matrix $B=\left[b_{i j}\right]$.

In the above notation the sytem of ordinary differential equations

$$
d y_{i} / d x=\sum_{j=1}^{n} b_{i j} y_{j}+g^{i}\left(x, y_{1}, \ldots, y_{n}\right) \quad(i=1,2, \ldots, n)
$$


has the following form:

$d y / d x=B y+g(x, y), \quad$ where $\quad g(x, y)=\left(g^{1}(x, y), \ldots, g^{n}(x, y)\right)$.

2. Definition 1. We say that the formal series $\sum_{i=0}^{\infty} u_{l}(Y)$ is of type $W$ if $u_{0}(Y)=$ const and, for each positive integer $l, u_{l}(Y)$ is a homogeneous polynomial of order $l$ in variables $y_{0}, y_{1}, \ldots, y_{n}$, namely

$$
u_{0}(Y)=\text { const }, \quad u_{1}(Y)=a_{0} y_{0}+a_{1} y_{1}+\ldots+a_{n} y_{n} .
$$

The series of one variable $\sum_{l=0}^{\infty} v_{l}(x), \quad v_{0}(x)=$ const, $v_{l}(x)=b_{l} x^{l}$ $(l=1,2, \ldots)$ is a special case of the series of type $W$.

DeFinItion 2. Let $H(Y)$ be a function defined in a neighbourhood of point $O_{n+1}$. We say that the function $H(Y)$ possesses an asymptotic development of the type $W$ at point $O_{n+1}$ (which is written as)

$$
H(Y) \sim \sum_{l=0}^{\infty} u_{l}(Y)
$$

if for every non-negative integer $m$ the following relation holds:

$$
\lim _{Y \rightarrow O_{n+1}}|Y|^{-m}\left[H(Y)-\sum_{l=0}^{m} u_{l}(Y)\right]=0 \quad(m=0,1,2, \ldots) .
$$

This relation means that there exists a function $E_{m}(Y)$ continuous at $O_{n+1}$ which satisfies the condition $E_{m}\left(O_{n+1}\right)=0$ and that in a neighbourhood of point $O_{n+1}$ the following equality holds true:

$$
H(Y)-\sum_{l=0}^{m} u_{l}(Y)=|Y|^{m} E_{m}(Y) \text {. }
$$

If on a neighbourhood of $O_{n+1}$ we have $u_{l}(Y) \equiv 0(l=0,1,2, \ldots)$, we write relation (1.2) briefly as

$$
H(\bar{Y}) \sim 0
$$

and we say that the function $H(Y)$ possesses a zero asymptotic development of the type $W$ at point $O_{n+1}$.

The sequence of asymptotic developments of functions $\boldsymbol{S}_{i}(\boldsymbol{Y})$ $(i=1,2, \ldots, n)$ is said to be an asymptotic development of the vector function $S(Y)=\left(S_{1}(Y), S_{2}(Y), \ldots, S_{n}(Y)\right)$.

Remark 1. Every function $F(Y)$ possesses at most one asymptotic development of the type $W$ at point $O_{n+1}$. 
3. Consider the system of ordinary differential equations

$$
d y / d x=A(x, y), \quad A(x, y)=\left(A^{1}(x, y), \ldots, A^{n}(x, y)\right) .
$$

Let the function $A(x, y)$ be continuous on an open domain $D \subset E_{n+1}$.

Suppose that through each point of domain $D$ there passes exactly one integral of system (1.4). We denote by $y=\psi(x ; P)$ the integral of system (1.4) passing through the point $P \in D, P=\left(p_{0}, p_{1}, \ldots, p_{n}\right)$, $\left(\psi\left(p_{0} ; P\right)=\left(p_{1}, \ldots, p_{n}\right)\right)$.

We put

$$
I(x, P)=(x, \psi(x ; P)) \text {. }
$$

Let us denote by $\omega$ a certain domain contained in $D$ an by $F$ the boundary of the domain $\omega$ in $D$ (with respect to $D$ ).

By $\widetilde{\omega}$ we mean the set

$$
\widetilde{\omega}=D \backslash(\omega \cup F) .
$$

Let $P \in F$. If there exists an $\varepsilon>0$ such that

$$
I\left(\left(p_{0}-\varepsilon, p_{0}\right), P\right) \subset \omega,
$$

then $P$ is said to be a point of egress from domain $\omega$ with respect to system (1.4) and $D[7]$.

If $P$ is a point of egress from domain $\omega$ with respect to system (1.4) (and $D$ ) and, moreover, if there exists a number $\delta>0$ such that

$$
I\left(\left(p_{0}, p_{0}+\delta\right), P\right) \subset \widetilde{\omega},
$$

then $P$ is said to be a point of strict egress from $\omega$ with respect to system (1.4) and $D$.

Remark 2. It follows from the definition above that the point of strict egress from a domain $\omega$ with respect to system (1.4) and $D$ is a point of egress from domain $\omega$ with respect to system (1.4) and $D$.

Let $g(x, y)$ be a real function of real variables $x, y$ on a domain $D$, possessing continuous partial derivatives with respect to $y$ and bounded left-hand and right-hand partial derivatives with respect to $x$.

Let us put

$$
\varphi(x)=g(I(x, P))=g(x, \psi(x, P)) .
$$

Right-hand derivative $D_{+} \varphi(x)$ (left-hand derivative $D_{-} \varphi(x)$ ) of the function $\varphi(x)$ at $x=p_{0}$ is said to be the right-hand (left-hand) inclination of the function $g(x, y)$ at the point $P$ with respect to the system (1.4) and is denoted by the symbol $\left.S_{+} g\right|_{P}\left(\left.S_{-} g\right|_{P}\right)$.

From (1.4) we infer that

$$
\left.S_{+} g\right|_{P}=\frac{\partial}{\partial x_{+}} g(P)+\sum_{j=1}^{n} g_{y_{j}}(P) A^{j}(P) \quad\left(\left.S_{-} g\right|_{P}=\frac{\partial}{\partial x_{-}} g(P)+\sum_{j=1}^{n} g_{y_{j}}(P) A^{j}(P)\right) .
$$


Now let us define the sets

$$
\begin{aligned}
\omega & =\{(x, y) \in D: l(x, y)<0, m(x, y)<0\}, \\
L & =\{(x, y) \in D: l(x, y)=0, m(x, y) \leqslant 0\}, \\
M & =\{(x, y) \in D: m(x, y)=0, l(x, y) \leqslant 0\},
\end{aligned}
$$

where functions $l(x, y), m(x, y)$ are continuous in the domain $D$ and possess continuous partial derivatives in $y$ and bounded left-hand and right-hand partial derivatives in $x$.

The union of sets $L, M$ contains the boundary Fr $\omega$ of the domain $\omega$ in $D$.

Let us consider a point $P \in M \cap$ Fr $\omega$. It is easy to see that if $\left.S_{-} m\right|_{P}<0$, then $P$ is not a point of egress from the domain $\omega$ with respect to (1.4) and $D$. Similarly if $P \in(L \backslash M) \cap \operatorname{Fr} \omega$ and $\left.S_{+} l\right|_{P}>0,\left.s_{-} l\right|_{P}>0$, then $P$ is a point of strict egress from the domain $\omega$ with respect to the system (1.4) and $D$.

\$ 2. LEMMA 1. If

$$
F^{i}(Y) \sim \sum_{l=0}^{\infty} u_{i}^{i}(Y) \quad(i=1,2, \ldots, q) \quad \text { and } \quad F(Y)=\sum_{i=1}^{q} F^{i}(Y) b_{i},
$$

where $b_{1}, b_{2}, \ldots, b_{q}$ are arbitrary numbers, then

$$
F(Y) \sim \sum_{l=0}^{\infty} u_{l}(Y)
$$

where $u_{l}(Y)=\sum_{i=1}^{q} b_{i} u_{l}^{i}(Y)$.

The proof of the lemma is evident.

LEMмA 2. If the function $F(Y)$ possesses a zero asymptotic development of the type $W$ at the point $O_{n+1}$ and function $\Phi(Z)$ satisfies the inequality

$$
|\Phi(Z)| \leqslant N|Z|, \quad Z=\left(z_{0}, z_{1}, \ldots, z_{n}\right)
$$

on a neighbourhood of $O_{n+1}$, then the function $f(Z)=F(\Phi(Z))$ possesses a zero asymptotic development of the type $W$ at the point $O_{n+1}$.

Proof. We have

$$
F(Y)=|Y|^{m} E_{m}(Y) \quad(m=0,1,2, \ldots),
$$

where functions $E_{m}(Y)$ are continuous at $O_{n+1}$ and tend to zero as $Y \rightarrow O_{n+1}$.

Substituting $\Phi(Z)$ for $Y$ in (2.2) we obtain $f(Z)=F(\Phi(Z))$ $=|\Phi(Z)|^{m} E_{m}(\Phi(Z))$. Taking into consideration (2.1) we have

$$
|f(Z)| \leqslant C|Z|^{m}\left|E_{m}(\Phi(Z))\right| \quad(m=0,1,2, \ldots),
$$


where $C$ is a certain constant. Hence

$$
|f(Z)||Z|^{-m} \leqslant C\left|E_{m}(\Phi(Z))\right| \quad(m=0,1,2, \ldots) .
$$

Therefore we have

$$
\lim _{Z \rightarrow O_{n+1}}|Z|^{-m}|f(Z)|=0 \quad(m=0,1,2, \ldots),
$$

because by (2.1) $\Phi(Z) \rightarrow 0$ as $Z \rightarrow O_{n+1}$.

Remark 3. If a real function $F(Y)$ of a real variable $Y$ belongs to the class $C^{\infty}$ on a neighbourhood of $O_{n+1}$, then it has an asymptotic development of the type $W$ at $O_{n+1}, F(Y) \sim \sum_{l=0}^{\infty} u_{l}(Y)$, where $\sum_{l=0}^{\infty} u_{l}(Y)$ is the Taylor series of $F(Y)$ at $O_{n+1}$.

LEMma 3. Let $u_{0}(Y), u_{1}(Y), u_{2}(Y), \ldots$ be a sequence of homogeneous polynomials, where $u_{l}(Y)$ is of order $l(l=0,1,2, \ldots)$.

Then there exists a function $H(Y)$ of class $C^{\infty}$ on a neighbourhood of $O_{n+1}$ such that

$$
H(Y) \sim \sum_{l=0}^{\infty} u_{l}(Y) .
$$

This lemma follows from Whitney's theorem [8].

LEMMA 4. Consider the system of ordinary differential equations

$$
|x|^{r} d z / d x=A z+\eta(x, z) z+R^{*}(x, z),
$$

where $r$ is an integer, $A$ is $a n \times n$ constant matrix and $\eta(x, z)=\left[\eta_{i j}\right]$ is $n \times n$ matrix function, where $\eta_{i j} \rightarrow 0$ as $x \nearrow 0, z \rightarrow O_{n}(i, j=1,2, \ldots, n)$.

(The symbol $x \nearrow 0$ means that $x \rightarrow 0$ and $x<0$.)

The vector function $R^{*}(x, z)$ possesses a zero asymptotic development of the type $W$ at the point $O_{n+1}$.

Let $\lambda_{1}, \lambda_{2}, \ldots, \lambda_{n}$ be the characteristic roots of the matrix $A$ and let $a_{q}=\operatorname{re} \lambda_{q}, \beta_{q}=\operatorname{im} \lambda_{q}, q=1,2, \ldots, n$.

There exists a linear non-singular transformation $\mathfrak{G}: u=T z$, which transforms (2.3) into the system

$$
|x|^{r} d u / d x=B u+\gamma(x, u) u+R^{* *}(x, u),
$$

where $B$ is a matrix of order $n$ of the form

$$
B=\left[\begin{array}{llll}
B_{1} & & & 0 \\
& B_{2} & & \\
& & \ddots & \\
0 & & B_{8}
\end{array}\right],
$$


$B_{p}(p=1,2, \ldots, s)$ is a square matrix of one of the following forms:

$$
\begin{aligned}
& {\left[\begin{array}{cccccc}
a_{p} & \delta_{p} & 0 & 0 & \ldots & 0 \\
0 & a_{p} & \delta_{p} & 0 & \ldots & 0 \\
. & \cdot & . & . & \ldots & . \\
0 & 0 & 0 & 0 & \ldots & a_{p}
\end{array}\right],} \\
& {\left[\begin{array}{cccccccccc}
a_{p} & \beta_{p} & \delta_{p} & 0 & 0 & 0 & 0 & \ldots & 0 & 0 \\
-\beta_{p} & a_{p} & 0 & \delta_{p} & 0 & 0 & 0 & \ldots & 0 & 0 \\
0 & 0 & a_{p} & \beta_{p} & \delta_{p} & 0 & 0 & \ldots & 0 & 0 \\
0 & 0 & -\beta_{p} & a_{p} & 0 & \delta_{p} & 0 & \ldots & 0 & 0 \\
. & . & . & . & . & . & . & \ldots & . & . \\
0 & 0 & 0 & 0 & 0 & 0 & 0 & \ldots & a_{p} & \beta_{p} \\
0 & 0 & 0 & 0 & 0 & 0 & 0 & \ldots & -\beta_{p} & a_{p}
\end{array}\right],}
\end{aligned}
$$

$\delta_{p}$ is arbitrary positive number, $\gamma(x, u)=\left[\gamma_{i j}(x, u)\right], \gamma_{i j}(x, u) \rightarrow 0$ as $x \nearrow 0$, $u \rightarrow O_{n}(i, j=1,2, \ldots, n)$ and $R^{* *}(x, u)$ possesses $a$ zero asymptotic development of the type $W$ at $O_{n+1}$.

Remark 4. We can easily show that there exists a finite number $\beta_{0}$ such that

$$
(B u) u \geqslant \beta_{0}|u|^{2}
$$

and there exists a function $e(x, u), e(x, u) \rightarrow 0$ as $x \nearrow 0, u \rightarrow O_{n}$ such that

$$
(\gamma(x, u) u) u \geqslant-|e(x, u)||u|^{2}
$$

moreover, for an arbitrary constant $\boldsymbol{A}_{m}$, there is a function $\zeta_{m}(x), \zeta_{m}(x) \rightarrow 0$ as $x \nearrow 0$, such that

$$
R^{* *}(x, u) u \geqslant-\left|\zeta_{m}(x)\right||x|^{2 m+2}
$$

for $|u| \leqslant A_{m}|x|, m$ is an integer, $m \geqslant 1$.

LEMMA 5. Assume that re $\lambda_{q}=a_{q} \neq 0(q=1,2, \ldots, n)$ and let

$$
a_{1} \geqslant a_{2} \geqslant \ldots \geqslant a_{l}>0>a_{l+1} \geqslant \ldots \geqslant a_{n},
$$

then there exists a linear non-singular transformation $\mathfrak{G}: u=T z$, which transforms (2.3) into the system

$$
\begin{gathered}
|x|^{r} d v / d x=G^{1} v+\Gamma^{1}(x, u) u+R^{1}(x, u), \\
|x|^{r} d w / d x=G^{2} w+\Gamma^{2}(x, u) u+R^{2}(x, u),
\end{gathered}
$$

where $u=(v, w), v=\left(u_{1}, u_{2}, \ldots, u_{l}\right), w=\left(u_{l+1}, u_{l+2}, \ldots, u_{n}\right), G^{1}, G^{2}$ are $l \times l$ and $(n-l) \times(n-l)$ matrices respectively, having the form

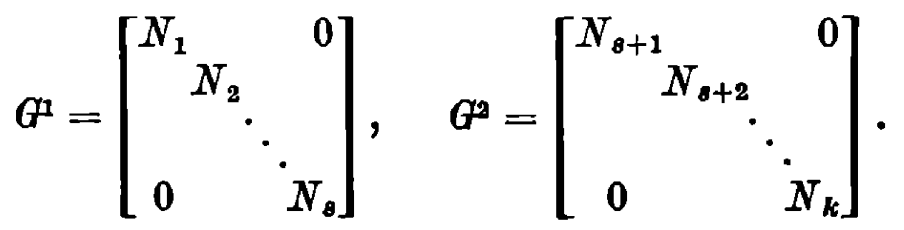


By $N_{p}(p=1,2, \ldots, k)$ we denote a matrix having one of the following forms:

$$
\left[\begin{array}{cccccccccc}
a_{p} & \beta_{p} & \delta & 0 & 0 & 0 & 0 & \ldots & 0 & 0 \\
-\beta_{p} & a_{p} & 0 & \delta & 0 & 0 & 0 & \ldots & 0 & 0 \\
0 & 0 & a_{p} & \beta_{p} & \delta & 0 & 0 & \ldots & 0 & 0 \\
0 & 0 & -\beta_{p} & a_{p} & 0 & \delta & 0 & \ldots & 0 & 0 \\
. & . & . & . & . & . & . & \ldots & . & . \\
0 & 0 & 0 & 0 & 0 & 0 & 0 & \ldots & a_{p} & \beta_{p} \\
0 & 0 & 0 & 0 & 0 & 0 & 0 & \ldots & -\beta_{p} & a_{p}
\end{array}\right] ; \quad\left[\begin{array}{cccccc}
a_{p} & \delta & 0 & 0 & \ldots & 0 \\
0 & a_{p} & \delta & 0 & \ldots & 0 \\
. & . & . & . & \ldots & . \\
0 & 0 & 0 & 0 & \ldots & a_{p}
\end{array}\right] .
$$

Number $\delta$ can be chosen so as to satisfy the inequality

$$
|\delta|<(2 n)^{-1} \min \left(a_{1}, \ldots, a_{l},-a_{l+1}, \ldots,-a_{n}\right) .
$$

We have $\Gamma(x, u)=\left(\Gamma^{1}(x, u), \Gamma^{2}(x, u)\right) \rightarrow 0$ as $x \nearrow 0, u \rightarrow O_{n}, R^{* *}(x, u)$ $=\left(R^{1}(x, u), R^{2}(x, u)\right)$ is a vector possessing a zero asymptotic development of the type $W$ at point $O_{n+1}$.

Remark 5. With the help of relations (2.8), (2.10) we can easily show that there exist constants $a>0, \beta<0$ such that

$$
\left(G^{1} v\right) v \geqslant \alpha|v|^{2}, \quad\left(G^{2} w\right) w \leqslant \beta|w|^{2}
$$

and there exists a function $e(x, u), e(x, u) \rightarrow 0$ as $x \nearrow 0, u \rightarrow O_{n}$ such that

$$
\begin{aligned}
& \Gamma^{1}(x, u) v \geqslant-|e(x, u)|\left(|v|^{2}+|w|^{2}\right), \\
& \Gamma^{2}(x, u) w \leqslant+\mid e(x, u) !\left(|v|^{2}+|w|^{2}\right) ;
\end{aligned}
$$

moreover, for an arbitrary constant $A_{m}$ there is a function $\gamma_{m}(x), \gamma_{m}(x) \rightarrow 0$ as $x \nearrow 0$, such that

$$
\begin{aligned}
R^{1}(x, u) v & \geqslant-\left|\gamma_{m}(x)\right||x|^{2 m+2}, \\
R^{2}(x, u) w & \leqslant\left|\gamma_{m}(x)\right||x|^{2 m+2}
\end{aligned}
$$

for $|u| \leqslant A_{m}|x|^{m}$, where $m \geqslant 1$ is an integer.

\$3. We now give the main results of this paper.

Consider the system of $n$ ordinary differential equations

$$
|x|^{r} d y / d x=f(x, y), \quad f(x, y)=\left(f^{1}(x, y), \ldots, f^{n}(x, y)\right)
$$

where $r$ is an integer.

We shall now formulate the following assumptions.

1) Vector function $f(x, y)$ is continuous on a neighbourhood $\Omega$ of the origin $O_{n+1}, f\left(O_{n+1}\right)=O_{n}$ and possesses an asymptotic development of the type $W$ at point $O_{n+1}$,

$$
f^{i}(x, y) \sim \sum_{l=0}^{\infty} u_{l}^{i}(x, y) \quad(i=1,2, \ldots, n)
$$


where

$$
u_{0}^{\prime}(x, y)=0, \quad u_{1}^{i}(x, y)=a_{i 0} x+a_{i 1} y_{1}+\ldots+a_{i n} y_{n}
$$

for $i=1,2, \ldots, n$.

2) Through each point of the domain $\Omega_{1}=\Omega \backslash \Pi$ (II denotes the plane $x=0$ ) passes exactly one integral of the system (3.1).

3) The real parts of all characteristic roots $\lambda_{1}, \ldots, \lambda_{n}$ of $n \times n$ matrix $A=\left[a_{i j}\right]$ are different from zero, i.e.

$$
\text { re } \lambda_{q}=a_{q} \neq 0, \quad q=1,2, \ldots, n .
$$

In order to fix attention we put

$$
a_{1} \geqslant a_{2} \geqslant \ldots a_{l}>0>a_{l+1} \geqslant \ldots \geqslant a_{n} .
$$

4) Functions $f^{i}(x, y), i=1,2, \ldots, n$, have first partial derivatives with respect to $y_{1}, \ldots, y_{n}$ in $\Omega_{1}$ and they have finite limits as $x \nearrow 0, y \rightarrow O_{n}$.

We shall study separately the properties of the integrals of (3.1) in the cases

a) $r=0$ (Theorem 1),

b) $r=1$ (Theorem 2),

c) $r \geqslant 2$ (Theorems $3,4,5,6,7$ ).

The case $r<0$ reduces trivially to the case $r=0$.

THEOREM 1. Assume 1) and suppose that through each point of domain $\Omega$ passes exactly one integral of the system

$$
d y / d x=f(x, y) \text {. }
$$

Then the integral of the system passing through the point $O_{n+1}$ possesses an asymptotic development at $x=0$.

THEOREM 2. For $r=1$, under assumptions 1), 2) and under the assumption that

$$
\operatorname{Det}[A+m I] \neq 0
$$

for every non-negative integer $m$, the system (3.1) possesses a solution tending to $O_{n}$ as $x \rightarrow 0$ and having an asymptotic development at $x=0$.

ThEOREM 3. Under assumptions 1), 2), 3) the system (3.1) $(r \geqslant 2)$ possesses a solution tending to $O_{n}$ as $x \nearrow_{0}$ and having an asymptotic development at $x=0$, moreover, if $\alpha_{\alpha}$ are not all positive, there are infinitely many integrals of (3.1) possessing the same development at $x=0$.

TheоREM 4. Under assumptions 1), 2) if

$$
a_{1} \leqslant a_{2} \leqslant \ldots \leqslant a_{n}<0,
$$

then all integrals of the system (3.1) $(r \geqslant 2)$ satisfying $|y(x)| \leqslant M|x|$, where $M$ is a positive constant, on a neighbourhood of the point $x=0$ possess the same asymptotic development at $x=0$. 
THEOREM 5. Under assumptions 1), 4) if

$$
a_{1} \geqslant a_{2} \geqslant \ldots \geqslant a_{n}>0,
$$

then the system (3.1) $(r \geqslant 2)$ possesses exactly one integral tending to $O_{n}$ as $x \nearrow_{0}$ and this integral possesses an asymptotic development at $x=0$.

THEOREM 6. Under assumptions 1), 3), 4) there exists such a neighbourhood $N$ of the origin that the set of points lying on the integrals of $(3.1)(r \geqslant 2)$ remaining in $N$ for $x \nearrow_{0}$ is a Lipschitzian $(n-l+1)$-dimensional manifold and each integral of the system (3.1) $(r \geqslant 2)$ remaining in $N$ for $x \nearrow 0$ possesses the same asymptotic development at $x=0$.

\section{§4. Proof of Theorem 1.}

1. Let $f(x, y) \sim \sum_{l=0}^{\infty} u_{l}(x, y)$. In virtue of Lemma 3 for polynomials $u^{i}(x, y)(i=1,2, \ldots, n)$ appearing in the system $(3.1)(r \leqslant 0)$ there exists a vector function. $H(x, y)=\left(H^{1}(x, y), \ldots, H^{n}(x, y)\right)$ of class $C^{\infty}$ defined on a neighbourhood $\Omega$ of $O_{n+1}$ such that $H^{i}(x, y) \sim \sum_{l=0}^{\infty} u_{l}^{i}(x, y), i=1,2, \ldots, n$.

From assumption 1) it is easily seen that

$$
H^{i}\left(O_{n+1}\right)=0 \quad(i=1,2, \ldots, n) .
$$

With the aid of functions $H^{i}(x, y)$ we may otherwise write $(3.1)(r \leqslant 0)$ in the form

$$
d y / d x=\boldsymbol{H}(x, y)+g(x, y)
$$

where $g(x, y)=\left(f^{1}(x, y)-H^{1}(x, y), \ldots, f^{n}(x, y)-H^{n}(x, y)\right), g(x, y) \sim 0$.

Let $y=\bar{y}(x)$ be the solution of the system $d y / d x=H(x, y)$ with the initial condition $\bar{y}(0)=O_{n}$.

2. We shall use the transformation

$$
z_{i}=y_{i}-\bar{y}_{i}(x) \quad(i=1,2, \ldots, n) .
$$

Then (4.2) takes the form

$$
d z / d x=H(x, z+\bar{y}(x))+g(x, z+\bar{y}(x))-\bar{y}^{\prime}(x)
$$

or

$$
d z / d x=H(x, z+\bar{y}(x))-B(x, \bar{y}(x))+g(x, z+\bar{y}(x)) .
$$

We have

$$
H(x, z+\bar{y}(x))-H(x, \bar{y}(x))=H_{\nu}(x, z t+\tilde{y}(x)) z,
$$

where $0<t<1$ and $H_{y}=\left[H_{y_{j}}^{t}(x, y)\right]$ denotes the Jacobian matrix of $\boldsymbol{H}(\boldsymbol{x}, \boldsymbol{y})$. 

obtain

On the other hand $H_{y_{i}}^{i}\left(O_{n+1}\right)=a_{i j}, i, j=1,2, \ldots, n$. Hence we

$$
H(x, z+\bar{y}(x))-H(x, \bar{y}(x))=(A+\eta(x, z)) z .
$$

From relations (4.5), (4.6) we may write the system (4.4) in the form $\overline{(4.4)}$

$$
d z / d x=A z+\eta(x, z) z+G(x, z),
$$

where $\eta(x, z)=\left[\eta_{i j}(x, z)\right]$ is a $n \times n$ matrix, $G(x, z)=g(x, z+\bar{y}(x))$.

Therefore the system $\overline{(4.4)}$ is of the same type as (2.3). On the basis of Lemma 4, a linear non-singular transformation $\mathcal{G}$ changes $\overline{(4.4)}$ into the system

$$
d u / d x=B u+\gamma(x, u) u+R^{* *}(x, u),
$$

where the matrix $B$ and the vectors $\gamma(x, u), R^{* *}(x, u)$ have the same meaning as in (2.4).

Transformation (4.3) changes $\Omega$ into a domain $\bar{\Omega}$.

3. Now we shall construct a sequence of auxiliary sets $S_{1}, S_{2}, \ldots$

Let us consider a number $A_{1}>0$ and a number $\xi_{0} \in(-1,0)$ such that the set $\bar{D}_{0}\left(\xi_{0}, A_{1}\right)=\left\{(x, u): x \in\left(\xi_{0}, 0\right),|u|^{2}-A_{1}^{2} x^{2}<0\right\}$ is contained in $\bar{\Omega}$.

Consider the expression

$$
\frac{d}{d x}\left(|u|^{2}-A_{1}^{2} x^{2}\right)=2 u \frac{d u}{d x}-2 A_{1}^{2} x
$$

for $|u|^{2}=A_{1}^{2} x^{2}, \xi_{0} \leqslant x$, where $d u / d x$ denotes the right-hand members of the system (4.7).

Now

$$
2 u \frac{d u}{d x}-2 A_{1}^{2} x=2\left[(B u) u+(\gamma(x, u) u) u+R^{* *}(x, u) u\right]-2 A_{1}^{2} x .
$$

Therefore it is easy to see that there exists a number $\xi_{1}: \xi_{0}<\xi_{1}<0$, that

$$
\frac{d}{d x}\left(|u|^{2}-A_{1}^{2} x^{2}\right)>0 \quad \text { for } \quad|u|^{2}=A_{1}^{2} x^{2}, \quad \xi_{1} \leqslant x<0
$$

or that the set $S_{1}=\left\{(x, u): \xi_{1} \leqslant x<0,|u|^{2}-A_{1}^{2} x^{2}=0\right\}$ has the property that (4.8) is satisfied on $S_{1}$.

Let us take into considerations the set

$$
S_{m}=\left\{(x, u): \xi_{m}<x<0,|u|^{2}-A_{m}^{2} x^{2 m}=0\right\},
$$

where $A_{m}>0$ is a certain constant, $\xi_{1}<\xi_{m}<0$. Suppose now that

$$
\frac{d}{d x}\left(|u|^{2}-A_{m}^{2} x^{2 m}\right)>0 \quad \text { on } S_{m} \quad(m \geqslant 1),
$$

where $d u / d x$ is replaced by the right-hand members of (4.7). 
Let $\xi: \xi_{m}<\xi<0$ be an arbitrary fixed number and let us consider the expression

$$
\begin{aligned}
& \frac{d}{d x}\left(|u|^{2}-\left(A_{m}^{2} / \xi^{2}\right) x^{2 m+2}\right) \\
& \quad=2 u \frac{d u}{d x}-(2 m+2)\left(A_{m}^{2} / \xi^{2}\right) x^{2 m+1} \\
& \quad=2\left[(B u) u+(\gamma(x, u) u) u+R^{* *}(x, u) u\right]-(2 m+2)\left(A_{m}^{2} / \xi^{2}\right) x^{2 m+1}
\end{aligned}
$$

for $|u|^{2}=\left(A_{m}^{2} / \xi^{2}\right) x^{2 m+2}, \xi \leqslant x<0$.

We have $|u|=\left(A_{m} /|\xi|\right)|x|^{m+1} \leqslant A_{m}|x|^{m}$, because $|x| \xi \mid \leqslant 1$. Hence with the aid of inequalities (2.5), (2.6), (2.7) we obtain the inequality

$$
\begin{aligned}
2[(B u) & \left.u+(\gamma(x, u) u) u+R^{* *}(x, u) u\right]-(2 m+2)\left(A_{m}^{2} / \xi^{2}\right) x^{2 m+1} \\
\leqslant & 2\left[\beta_{0}|u|^{2}-|e(x, u)||u|^{2}-\left|\zeta_{m}(x)\right| x^{2 m+2}\right]-(2 m+2)\left(A_{m}^{2} / \xi^{2}\right) x^{2 m+1} \\
& =2\left(A_{m}^{2} / \xi^{2}\right) x^{2 m+1}\left[|x|\left(\beta_{0}+\delta_{m}(x)+\Delta_{m}(x)\left(\xi^{2} / A_{m}^{2}\right)\right)+(m+1)\right]
\end{aligned}
$$

where $\delta_{m}(x), \Delta_{m}(x)$ are independent of $\xi$ and tend to zero as $x / 0$.

There is a number $\xi_{m+1}, \xi_{m}<\xi_{m+1}<0$, independent of $\xi$ such that

$$
|x|\left(\beta_{0}+\delta_{m}(x)+\Delta_{m}(x)\left(\xi^{2} / A_{m}^{2}\right)\right)+(m+1)>0
$$

for $\xi_{m+1} \leqslant x<0$.

Finally we have the following inequality

$$
\frac{d}{d x}\left(|u|^{2}-\left(A_{m}^{2} / \xi^{2}\right) x^{2 m+2}\right)>0 \quad \text { for } \quad|u|^{2}=\left(A_{m}^{2} / \xi^{2}\right) x^{2 m+2}, \xi_{m+1} \leqslant x<0,
$$

where $d u / d x$ is replaced by the right-hand membres of (4.7) and $\xi$ is such a fixed number that $\xi_{m}<\xi<0$.

Therefore, in particular, the following inequality also holds:

$$
\begin{aligned}
& \frac{d}{d x}\left(|u|^{2}-\left(A_{m}^{2} / \xi_{m+1}^{2}\right) x^{2 m+2}\right)>0 \\
& \text { for } \quad|u|^{2}=\left(A_{m}^{2} / \xi_{m+1}^{2}\right) x^{2 m+2}, \xi_{m+1} \leqslant x<0,
\end{aligned}
$$

where $d u / d x$ is replaced by the right-hand members of (4.7).

We introduce the notation $A_{m+1}=-\left(A_{m} / \xi_{m+1}\right)\left(A_{m}>0\right)$.

It can be seen that polynomials $A_{m+1}(-x)^{m+1}, A_{m}(-x)^{m}$ have the same value at $x=\xi_{m+1}$.

With the aid of (4.10) we obtain the inequality

$$
\frac{d}{d x}\left(|u|^{2}-A_{m+1} x^{2 m+2}\right)>0 \quad \text { on } S_{m+1}
$$

where $d u / d x$ is replaced by the right-hand members of the system (4.7). 
We have thus shown that there exists a sequence $\left\{\xi_{m}\right\}$,

$$
-1<\xi_{m}<0, \quad \xi_{m}<\xi_{m+1}(m=0,1,2, \ldots), \quad \lim _{m \rightarrow \infty} \xi_{m}=0
$$

that the inequality (4.9) holds for every natural number $m$.

For the sequence (4.11) we define a function $l\left(x,\left\{\xi_{m}\right\}\right)$ in the following way:

$l\left(x,\left\{\xi_{m}\right\}\right)=(-1)^{m} A_{m} x^{m} \quad$ for $\quad x \in\left\langle\xi_{m-1}, \xi_{m}\right\rangle \quad(m=2,3, \ldots)$.

It is easy to see that the function $l\left(x,\left\{\xi_{m}\right\}\right)$ is defined, continuous and possesses a left-hand and a right-hand derivative in the whole interval $\left\langle\xi_{1}, 0\right\rangle$; moreover,

$$
l\left(x,\left\{\xi_{m}\right\}\right) \sim 0+0 x+0 x^{2}+\ldots \quad \text { at } x=0 .
$$

4. Let us introduce the notation

$$
\begin{gathered}
L(x, u)=|u|^{2}-l^{2}\left(x,\left\{\xi_{m}\right\}\right), \quad m(x, u)=\xi_{1}-x, \\
\widetilde{\Omega}=\bar{\Omega} \cap\left\{(x, u): u \in R^{n}, x<0\right\} .
\end{gathered}
$$

We define the domain $D$ as follows:

$$
D=\{(x, u) \in \widetilde{\Omega}, L(x, u)<0, m(x, u)<0\} .
$$

The boundary of the domain $D$ in $\widetilde{\Omega}$ is formed by the union of sets

$$
\begin{aligned}
& S^{(1)}=\{(x, u) \epsilon \widetilde{\Omega}, L(x, u)=0, m(x, u) \leqslant 0\}, \\
& S^{(2)}=\{(x, u) \epsilon \widetilde{\Omega}, L(x, u) \leqslant 0, m(x, u)=0\} .
\end{aligned}
$$

From the definition of functions $l\left(x,\left\{\xi_{m}\right\}\right), m(x, u)$ and inequalities (4.9) we have

$$
S_{-} L(x, u)>0, \quad S_{+} L(x, u)>0 \quad \text { on } S^{(1)}, \quad S_{-} m(x, u)<0 \quad \text { on } S^{(2)} \text {. }
$$

Hence it follows that points of $S^{(2)}$ are not points of egress from $D$ with respect to the system $(4.7)$ and to $\widetilde{\Omega}$, but points which belong to the set $S^{(1)} \backslash S^{(2)}$ are points of strict egress from $D$ with respect to (4.7) and to $\widetilde{\Omega}$. Therefore all points of egrees from the domain $D$ with respect to (4.7) and to $\widetilde{\Omega}$ are points of strict egress from the domain $D$ with respect to the system (4.7) and to $D$.

Consider a fixed number $x_{0}, \xi_{1}<x_{0}<0$ and let $Z=\left\{(x, u): x=x_{0}\right.$, $\left.|u|^{2}-l^{2}\left(x_{0},\left\{\xi_{m}\right\}\right) \leqslant 0\right\}$. It is not difficult to prove that $Z \subset D \cup\left(S^{(1)} \backslash S^{(2)}\right)$ and that $Z \cap\left(S^{(1)} \backslash S^{(2)}\right)$ is not a retract of the set $Z$, but is a retract of the set $S^{(1)} \backslash S^{(2)}$. Hence, on the basis of T. Ważewski's theorem [7], in the set $Z \backslash\left(S^{(1)} \backslash S^{(2)}\right)$ there exists at least one such point that the integral $u(x)$ of (4.7) starting from this point to the right remains in $D$, i.e.

$$
\left|u_{i}(x)\right|<l\left(x,\left\{\xi_{m}\right\}\right) \quad(i=1,2, \ldots, n) \quad \text { for } \quad x_{0} \leqslant x<0 .
$$


From the property (4.12) of the function $l\left(x,\left\{\xi_{m}\right\}\right)$ and from (4.13) the following relations follow:

$$
u_{i}(x) \sim 0+0 x+0 x^{2}+\ldots \quad(i=1,2, \ldots, n) .
$$

From the non-singularity of the linear transformation $\mathcal{T}$ it follows that (4.4) possesses an integral $z(x)=\left(z_{1}(x), \ldots, z_{n}(x)\right)$ having a zero asymptotic development at $x=0$ or

$$
z_{i}(x) \sim 0+0 x+0 x^{2}+\ldots \quad(i=1,2, \ldots, n) \quad \text { at } x=0 .
$$

With the aid of (4.3) we return to (3.1) and obtain an integral $y(x)$ $=\left(y_{1}(x), \ldots, y_{n}(x)\right)$ of the system (3.1) possessing an asymptotic development.

It means that (3.1) has an integral $y(x)$ tending to $O_{n}$ as $x \nearrow 0$ and possessing an asymptotic development at $x=0$. We have $y(0)=O_{n}$. From the fact that for each point of the neighbourhood $\Omega$ (3.1) has exactly one integral it follows that the integral passing through $O_{n+1}$ of the system (3.1) $(r \leqslant 0)$ possesses an asymptotic development at $x=0$.

Remark 6. Theorem 1 is analogous to the Cauchy-Kowalewsky theorem.

§5. Proof of Theorem $3(r \geqslant 2)$.

1. For the polynomials $u_{l}^{i}(x, y), i=1,2, \ldots, n$, appearing in (3.1) $(r \geqslant 2)$ there corresponds (Lemma 3 ) a vector function $H(x, y)$ $=\left(H^{1}(x, y), \ldots, H^{n}(x, y)\right)$ belonging to class $C^{\infty}$ in the neighbourhood $\Omega$ such that

$$
H^{i}(x, y) \sim \sum_{l=0}^{\infty} u_{l}^{i}(x, y) \quad(i=1,2, \ldots, n) .
$$

With the aid of (3.2) we have

$$
|A|=\left.\operatorname{Det}\left[H_{y_{j}}^{i}(x, y)\right]\right|_{(x, y) \Rightarrow O_{n+1}} \neq 0 \text {. }
$$

From assumption 1) and (5.1) we obtain

$$
H\left(O_{n+1}\right)=O_{n}, \quad H(0, y) \neq O_{n} \text { for } y \neq O_{n} .
$$

With the aid of $H(x, y)$ we may write (3.1) $(r \geqslant 2)$ in the form

$$
|x|^{r} d y / d x=H(x, y)+g(x, y) \text {, }
$$

where $g(x, y) \sim 0$.

2. We want to find a vector function $\varphi(x)=\left(\varphi_{1}(x), \ldots, \varphi_{n}(x)\right)$ in class $C^{\infty}$ on a certain neighbourhood of $x=0$

$$
(x, \varphi(x)) \in \Omega
$$


such that the functions $a_{i}(x)=|x|^{\tau} \varphi_{i}^{\prime}(x)-H^{\prime}(x, \varphi(x)), i=1, \ldots, n$, have a zero asymptotic development at $x=0$, i.e.

$$
\alpha_{i}^{(m)}(0)=0 \quad \text { for } \quad m=0,1,2, \ldots \quad(i=1,2, \ldots, n) .
$$

Passing to the limit for $a_{i}(x)$ as $x \nearrow 0$, we obtain

$$
a_{i}(0)=-H^{i}(0, \varphi(0)) \quad(i=1,2, \ldots, n) .
$$

From (5.2) we have

$$
\varphi(0)=O_{n}
$$

Let $\varphi^{(m)}(x)=\left(\varphi_{1}^{(m)}(x), \ldots, \varphi_{n}^{(m)}(x)\right), a^{(m)}(x)=\left(a_{1}^{(m)}(x), \ldots, a_{n}^{(m)}(x)\right)(m=1$, $2, \ldots), H_{x}(x, y)=\left(H_{x}^{1}(x, y), \ldots, H_{x}^{n}(x, y)\right)$, and let $H_{y}(x, y)=\left[H_{y_{j}}^{i}(x, y)\right]$ denote the Jacobian matrix of $H(x, y)$.

Differentiation of $a_{i}(x)$ yields

$$
\alpha^{\prime}(x)=-H(x, \varphi(x))+\left(|x|^{r}\right)^{\prime} \varphi^{\prime}(x)+|x|^{r} \varphi^{\prime \prime}(x)-H_{\nu}(x, \varphi(x)) \varphi(x) .
$$

It can be shown that the identities

$$
\begin{aligned}
a^{(m)}(x)=m\left(|x|^{r}\right)^{\prime} \varphi^{(m)}(x)-H_{y}(x, \varphi(x)) \varphi^{(m)}(x) & +|x|^{r} \varphi^{(m+1)}(x)+ \\
& +W_{m}\left(x, \varphi(x), \ldots, \varphi^{(m-1)}(x)\right)
\end{aligned}
$$

hold for every number $m$, where the vector function $W_{m}(x, \varphi(x), \ldots$ $\left.\ldots, \varphi^{(m-1)}(x)\right)$ is determined by $H(x, y)$ and its partial derivatives up to $m$ th order.

Using (5.6) and letting $\alpha^{(m)}(x)$ pass to the limit as $x \nearrow_{0}$ we obtain

$$
a^{(m)}(0)=-H_{\nu}\left(O_{n+1}\right) \varphi^{(m)}(0)+W_{m}\left(0, \varphi(0), \ldots, \varphi^{(m-1} \cdot(0)\right)=0
$$

for every natural $m$.

From (5.1) it follows that we can solve the system of linear equations (5.7) and let $\left(\gamma_{m 1}, \ldots, \gamma_{m n}\right)=\gamma_{m}=\varphi^{(m)}(0)$ be the solution of (5.7) for $m=1,2, \ldots$

In this way we obtain a sequence of vectors

$$
O_{n}=\gamma_{0}, \gamma_{1}, \gamma_{2}, \ldots
$$

Using Lemma 3 we obtain for the sequence (5.8) a vector function $\bar{y}(x)=\left(\bar{y}_{1}(x), \ldots, \bar{y}_{n}(x)\right)$ which is defined and belongs to class $C^{\infty}$ on a certain neighbourhood of $x=0$ and is such that

$$
\bar{y}(x) \sim 0+\gamma_{1} x+\gamma_{2} x^{2}+\ldots
$$

It is easy to see that for $\bar{y}(x)$ the required conditions $(5.4),(5.5)$ are satisfied. 
3. Let us introduce the change of variables

$$
z=y-\bar{y}(x) \text {. }
$$

Under this change, the system (5.3) takes the form

$$
|x|^{r} d z / d x=H(x, z+\bar{y}(x))+g(x, z+\bar{y}(x))-|x|^{r} \bar{y}^{\prime}(x) .
$$

With the aid of the properties of $H(x, y), \bar{y}(x)$ and using the meanvalue theorem we can write (5.11) in the form

$$
|x|^{r} d z / d x=A z+\eta(x, z) z+R^{*}(x, z),
$$

where functions $\eta(x, z), R^{*}(x, z)$ are of the same type as in (2.3).

In virtue of Lemma 5 , a linear non-singular transformation $\mathfrak{6}$ changes (5.12) into the system

$$
\begin{aligned}
& |x|^{\tau} d v / d x=G^{1} v+\Gamma^{1}(x, v, w) u+R^{1}(x, v, w), \\
& |x|^{\tau} d w / d x=G^{2} w+\Gamma^{2}(x, v, w) u+R^{2}(x, v, w),
\end{aligned}
$$

where matrices $G^{1}, G^{2}, \Gamma^{1}(x, v, w), \Gamma^{2}(x, v, w)$ and vector functions $R^{1}(x, v, w), R^{2}(x, v, w)$ have the same meaning as in system (2.9). Transformation $\mathcal{G}$ changes the domain $\Omega$ into a domain $\bar{\Omega}$.

4. Now we shall construct two sequences of auxiliary sets $S_{1}, S_{2}, \ldots$ and $Z_{1}, Z_{2}, \ldots$

For a given positive constant $A_{1}$ we choose a number $\xi_{0},-1<\xi_{0}<0$, such that the domain $D_{0}\left(\xi_{0}, A_{1}\right)=\left\{(x, v, w): \xi_{0}<x<0,|v|^{2}-A_{1}^{2} x^{2}<0\right.$, $\left.|w|^{2}-A_{1}^{2} x^{2}<0\right\}$ together with its boundary in $\bar{\Omega}_{1}$ is contained entirely in $\overline{\Omega_{1}}$.

Consider the expression

$$
|x|^{r} \frac{d}{d x}\left(|v|^{2}-A_{1}^{2} x^{2}\right)=2|x|^{r} v \frac{d v}{d x}-2 x A_{1}^{2}|x|^{r}
$$

for $|v|^{2}=A_{1}^{2} x^{2},|w|^{2} \leqslant A_{1}^{2} x^{2}$, where $|x|^{2} d v / d x$ is replaced by right-hand members of (5.13).

We have

$$
|x|^{r} \frac{d}{d x}\left(|v|^{2}-A_{1}^{2} x^{2}\right)=2\left[\left(G^{1} v\right) v+\left(\Gamma^{1}(x, v, w) u\right) v+R^{1}(x, v, w) v\right]-2 x A_{1}^{2}|x|^{r} .
$$

Since $|v|^{2}=A_{1}^{2} x^{2},|w|^{2} \leqslant A_{1}^{2} x^{2}$, we have

$$
|u| \leqslant 2 A_{1}|x| \text {. }
$$

By virtue of inequalities (2.11), (2.12), (2.13) appearing in Remark 5, we obtain

$$
\begin{aligned}
2\left[\left(G^{1} v\right) v+\left(\Gamma^{1}(x, v, w) u\right) v+\right. & \left.R^{1}(x, v, w) v\right]-2 x A_{1}^{2}|x|^{r} \\
& \left.\geqslant 2 A_{1}^{2} x^{2}\left[a-|e(x, u)|-\mid \gamma_{1}(x)\right]-|x|^{r} x^{-1}\right],
\end{aligned}
$$

where $e(x, u) \rightarrow 0$ as $x \nearrow 0, u \rightarrow O_{n}, \gamma_{1}(x) \rightarrow 0$ as $x \nearrow 0$. 
From (5.14) for $r \geqslant 2, a>0$ there exists a $t_{1}, \xi_{0}<t_{1}<0$ such that

$$
a-|e(x, u)|-\left|\gamma_{1}(x)\right|-|x|^{+} x^{-1} \geqslant a / 2 \quad \text { for } \quad t_{1} \leqslant x<0 .
$$

Therefore we have

$$
|x|^{r} \frac{d}{d x}\left(|v|^{2}-A_{1}^{2} x^{2}\right)>0 \quad \text { for } \quad|v|^{2}=A_{1}^{2} x^{2},|w|^{2} \leqslant A_{1}^{2} x^{2}, t_{1} \leqslant x<0
$$

Similarly we can show the existence of a constant $q_{1}, \xi_{0}<q_{1}<0$, such that

$$
|x|^{r} \frac{d}{d x}\left(|w|^{2}-A_{1}^{2} x^{2}\right)<0 \quad \text { for } \quad|v|^{2} \leqslant A_{1}^{2} x^{2},|w|^{2}=A_{1}^{2} x^{2}, q_{1} \leqslant x<0 .
$$

Let $\xi_{1}=\max \left(t_{1}, q_{1}\right)$; we see that

$$
\begin{aligned}
& \frac{d}{d x}\left(|v|^{2}-A_{1}^{2} x^{2}\right)>0 \quad \text { on } S_{1}, \\
& \frac{d}{d x}\left(|w|^{2}-A_{1}^{2} x^{2}\right)<0 \quad \text { on } Z_{1},
\end{aligned}
$$

where

$$
\begin{aligned}
& S_{1}=\left\{(x, v, w): \xi_{1}<x<0,|v|^{2}=A_{1}^{2} x^{2},|v|^{2} \leqslant A_{1}^{2} x^{2}\right\} \\
& Z_{1}=\left\{(x, v, w): \xi_{1}<x<0,|u|^{2}=A_{1}^{2} x^{2},|v|^{2} \leqslant A_{1}^{2} x^{2}\right\}
\end{aligned}
$$

and $|x|^{r} d v / d x,|x|^{r} d w / d x$ are replaced by the right-hand members of system (5.13).

As in $\S 4$, one can show that there exists a sequence of numbers $\left\{\xi_{m}\right\}$ such that

$$
-1<\xi_{m}<\xi_{m+1}<0 \quad(m=0,1,2, \ldots), \quad \lim _{m \rightarrow \infty} \xi_{m}=0,
$$

and the inequalities

$$
\begin{aligned}
& \frac{d}{d x}\left(|v|^{2}-A_{m}^{2} x^{2 m}\right)>0 \\
& \quad \text { on } S_{m}=\left\{(x, v, w): x \in\left(\xi_{m}, 0\right),|v|^{2}=A_{m}^{2} x^{2 m},|w|^{2} \leqslant A_{m}^{2} x^{2 m}\right\}, \\
& \frac{d}{d x}\left(|w|^{2}-A_{m}^{2} x^{2 m}\right)<0 \\
& \quad \text { on } Z_{m}=\left\{(x, v, w): x \in\left(\xi_{m}, 0\right),|w|^{2}=A_{m}^{2} x^{2 m},|v|^{2} \leqslant A_{m}^{2} x^{2 m}\right\}
\end{aligned}
$$

hold for every natural number $m$, where $|x|^{r} d v / d x,|x|^{r} d w / d x$ are replaced by the right-hand members of (5.13).

For sequence (5.15), we define a function $l\left(x,\left\{\xi_{m}\right\}\right)$ as in $\S 4$. 
It is easy to see that function $l\left(x,\left\{\xi_{m}\right\}\right)$ is defined and continuous in the whole interval $\left\langle\xi_{0}, 0\right)$; moreover, it possesses left-hand and righthand derivatives bounded in the interval $\left\langle\xi_{0}, 0\right)$ and the relation

$$
l\left(x,\left\{\xi_{m}\right\}\right) \sim 0+0 x+0 x^{2}+\ldots
$$

holds at $x=0$.

5. Let us introduce the notation

$$
\begin{gathered}
L(x, v, w)=|v|^{2}-l^{2}\left(x,\left\{\xi_{m}\right\}\right), \\
m^{1}(x, v, w)=|w|^{2}-l^{2}\left(x,\left\{\xi_{m}\right\}\right), \quad m^{2}(x, v, w)=\xi_{1}-x .
\end{gathered}
$$

We define

$$
\begin{array}{r}
D=\left\{(x, v, w):(x, v, w) \in \bar{\Omega}_{1}, L(x, v, w)<0, m^{1}(x, v, w)<0,\right. \\
\left.m^{2}(x, v, w)<0\right\} .
\end{array}
$$

The boundary of $D$ is formed by the union of sets

$$
\begin{aligned}
& \mathcal{S}^{(1)}=\left\{(x, v, w) \epsilon \bar{\Omega}_{1}, L(x, v, w)=0, m^{1}(x, v, w) \leqslant 0, m^{2}(x, v, w) \leqslant 0\right\}, \\
& S^{(2)}=\left\{(x, v, w) \epsilon \bar{\Omega}_{1}, L(x, v, w) \leqslant 0, m^{1}(x, v, w)=0, m^{2}(x, v, w) \leqslant 0\right\}, \\
& S^{(3)}=\left\{(x, v, w) \epsilon \bar{\Omega}_{1}, L(x, v, w) \leqslant 0, m^{1}(x, v, w) \leqslant 0, m^{2}(x, v, w)=0\right\} .
\end{aligned}
$$

In virtue of (5.16) it is easy to see that

$$
\begin{array}{rll}
S_{-} L(x, v, w)>0, & S_{+} L(x, v, w)>0 & \text { on } S^{(1)}, \\
S_{-} m^{1}(x, v, w)<0, & S_{+} m^{1}(x, v, w)<0 & \text { on } S^{(2)}, \\
S_{-} m^{2}(x, v, w)<0, & S_{+} m^{2}(x, v, w)<0 & \text { on } S^{(3)} .
\end{array}
$$

It follows that the points which belong to the set $S^{(2)} \cup S^{(3)}$ are not points of egress from the domain $D$ with respect to (5.13) and to $\bar{\Omega}_{1}$, but the points which belong to set $S^{(1)} \backslash\left(S^{(2)} \cup S^{(3)}\right)$ are points of strict egress from the domain $D$ with respect to system (5.13) and to $\bar{\Omega}_{1}$. Therefore all points of egress from the domain $D$ with respect to (5.13) and to $\overline{\Omega_{1}}$ are points of strict egress with respect to the system (5.13) and to $\vec{\Omega}_{1}$.

Let us consider an arbitrary fixed number $x_{0}$ and a vector $w_{0}=\left(u_{l+1}^{0}, \ldots, u_{n}^{0}\right)$ such that $\xi_{1}<x_{0}<0,\left|w_{0}\right|^{2}<l^{2}\left(x_{0},\left\{\xi_{m}\right\}\right)$. Let $Z=\{(x, v, w)$ : $\left.x=x_{0},|v|^{2}-l^{2}\left(x_{0},\left\{\xi_{m}\right\}\right) \leqslant 0, w=w_{0}\right\}$.

It is not difficult to prove that $Z \subset D \cup\left(S^{(1)} \backslash\left(S^{(2)} \cup S^{(3)}\right)\right)$ and that $Z \cap\left(S^{(1)} \backslash\left(S^{(2)} \cup S^{(3)}\right)\right)$ is not a retract of the set $Z$, but it is a retract of the set $\left(S^{(1)} \backslash\left(S^{(2)} \cup S^{(3)}\right)\right)$.

Hence with the aid of T. Ważewski's theorem [7] there exists at least one point which possesses the property that the integral $(v(x), w(x))$ of (5.13) starting from that point to the right remains in the domain $D$. 
Therefore we have

$$
\left|u_{i}(x)\right|<l\left(x,\left\{\xi_{m}\right\}\right) . \quad(i=1,2, \ldots, n) .
$$

By virtue of (5.18) and the property (5.17) of the function $l\left(x,\left\{\xi_{m}\right\}\right)$ it follows that $u_{i}(x) \sim 0+0 x+0 x^{2}+\ldots \quad(i=1,2, \ldots, n)$. By the nonsingularity of the linear transformation $\mathcal{G}$, we infer that (5.12) possesses an integral $z(x)=\left\{z_{1}(x), \ldots, z_{n}(x)\right)$ having a zero asymptotic development at $x=0$, i.e. $z_{i}(x) \sim 0+0 x+0 x^{2}+\ldots(i=1,2, \ldots, n)$.

We return to system (3.1) $(r \geqslant 2)$ by relation (5.10) and we obtain an integral $y(x)=\left(y_{1}(x), \ldots, y_{n}(x)\right)$ of $(3.1)(r \geqslant 2)$ possessing an asymptotic development. It means that the system (3.1) $(r \geqslant 2)$ has an integral tending to $O_{n}$ as $x \nearrow 0$ and possessing an asymptotic development at $x=0$.

Since the wector $w_{0}$ is taken arbitrarily, there are infinitely many integrals of system (3.1) $(r \geqslant 2)$ (except for the case $a_{1} \geqslant a_{2} \geqslant \ldots \geqslant a_{n}>0$ ) tending to $O_{n}$ as $x \nearrow 0$ and possessing the same asymptotic development at $x=0$.

\$6. Proof of Theorem 2. If $r=1$, then the system (3.1) has the form

$$
|x| d y / d x=f(x, y), \quad f(x, y)=\left(f^{1}(x, y), \ldots, f^{n}(x, y)\right) .
$$

1. We may write (6.1) in the form

$$
|x| d y / d x=H(x, y)+g(x, y),
$$

where $H^{i}(x, y)(i=1,2, \ldots, n)$ are functions of class $C^{\infty}$ in the neighbourhood $\Omega$ and are such that

$$
H^{i}(x, y) \sim \sum_{l=0}^{\infty} u_{l}^{i}(x, y) \quad(i=1,2, \ldots, n)
$$

and the vector function $g(x, y)$ possesses a zero asymptotic development of the type $W$ at $O_{n+1}$, or $g(x, y) \sim 0$.

2. We want to find a vector function $\varphi(x)=\left(\varphi_{1}(x), \ldots, \varphi_{n}(x)\right)$ which is defined and belongs to class $C^{\infty}$ on a neighbourhood of point $x=0$, $\varphi(0)=O_{n}$.

$$
(x, \varphi(x)) \in \Omega
$$

and is such that the vector function $a(x)=|x| \varphi^{\prime}(x)-H(x, \varphi(x))$ possesses a zero asymptotic development at $x=0$, or

$$
a_{i}(x) \sim 0+0 x+0 x^{2}+\ldots \quad(i=1,2, \ldots, n) .
$$

Passing to the limit with $a_{i}(x)$ we obtain

$$
\alpha(0)=H(0, \varphi(0))=O_{n} \text {. }
$$


From (6.5) with the aid of assumption (3.3), we can show that $\varphi(0)=O_{n}$. Let $\gamma_{0}=\varphi(0)$.

Differentiation of $a(x)$ yields:

$$
a^{\prime}(x)=|x| \varphi^{\prime \prime}(x)+(|x|)^{\prime} \varphi^{\prime}(x)-H_{y}(x, \varphi(x)) \varphi^{\prime}(x)-B_{x}(x, \varphi(x)) .
$$

Passing to the limit with $\alpha^{\prime}(x)$ we obtain

$$
H_{y}\left(O_{n+1}\right) \varphi^{\prime}(0)+\varphi^{\prime}(0)=-H_{x}\left(O_{n+1}\right) .
$$

It is a linear non-homogeneous system for $\varphi^{\prime}(0)$. From the assumption (3.3) we obtain $\left|H_{y}\left(O_{n+1}\right)+I\right| \neq 0$; therefore (6.6) has one and only one solution $\varphi^{\prime}(0)=\gamma_{1}$.

It can be shown that the identity

$$
\begin{aligned}
a^{(m)}(x)=|x| \varphi^{(m+1)}(x)+m(|x|)^{\prime} \varphi^{(m)}(x)-H_{y} & (x, \varphi(x)) \varphi^{(m)}(x)+ \\
& +W_{m}\left(x, \varphi^{\prime}(x), \ldots, \varphi^{(m-1)}(x)\right)
\end{aligned}
$$

holds for every natural $m$, where $W_{m}\left(x, \varphi(x), \ldots, \varphi^{(m-1)}(x)\right)$ is determined by the function $H(x, \varphi(x))$ and their partial derivatives up to $m$ th order.

Passing to the limit with $a^{(m)}(x)$ we have

$$
\left(H_{y}\left(O_{n+1}\right)+m I\right) \varphi^{(m)}(0)=W_{m}\left(0, \varphi(0), \ldots, \varphi^{(m-1)}(0)\right) .
$$

Hence with the aid of assumption (3.3) we can obtain $\varphi^{(m)}(0)=\gamma_{m}$.

Therefore we obtain a sequence of vectors

$$
O_{n}=\gamma_{0}, \gamma_{1}, \gamma_{2}, \ldots
$$

Using Lemma 3, we obtain a function $\bar{\varphi}(x)$ which is defined and belongs to class $C^{\infty}$ on a certain neighbourhood of $x=0$ and is such that

$$
\bar{\varphi}(x) \sim \gamma_{0}+\gamma_{1} x+\gamma_{2} x^{2}+\ldots
$$

It is easy to prove that the required conditions (6.3), (6.4) are satisfied for $\bar{\varphi}(x)$.

Introduce the change of variables

$$
z=y-\bar{\varphi}(x) \text {. }
$$

With the help of the linear non-singular transformation $\mathfrak{G}: u=T z$, $|T| \neq 0$ system (6.2) can be replaced by the system

$$
|x| d u / d x=B u+\gamma(x, u) u+R^{* *}(x, u),
$$

where matrices $B, \gamma(x, u)$ and vector $R^{* *}(x, u)$ are of the same type as in (2.4). The proof can be completed analogously to the proof of Theorem 3.

§ 7. Proof of Theorem $4\left(a_{1} \leqslant a_{2} \leqslant \ldots \leqslant a_{n}<0\right)$. Let us take into consideration a certain integral $u(x)$ of (6.13) satisfying

$$
|u(x)| \leqslant M|x|
$$

in a neighbourhood of $x=0$, where $M$ is a certain positive constant. 
Consider now the set $D$ defined in $\S 5$ with $\xi_{1}$ corresponding to $A_{1}>M$ and such that (7.1) is satisfied for $x=\xi_{1}$. In virtue of (7.1) integral $u(x)$ belongs to $D$ for $x=\xi_{1}$ and by (5.16) ( $S_{m}$ being empty) for $\xi_{1} \leqslant x<0$. Therefore $u(x)$ has the asymptotic development defined by (5.9) and the proof of Theorem 4 is complete.

§8. Proof of Theorem $5\left(r \geqslant 2, a_{1} \geqslant a_{2} \geqslant \ldots \geqslant a_{n}>0\right)$. Theorem 3 implies the existence of an integral of (3.1) tending to $O_{n}$ as $x \nearrow 0$.

Suppose that the system (3.1) $(r \geqslant 2)$ possesses two integrals $y^{1}(x)$, $y^{2}(x)$ such that

$$
y^{1}(x) \rightarrow O_{n}, \quad y^{2}(x) \rightarrow O_{n} \quad \text { as } x \nearrow 0 .
$$

In a certain neighbourhood of $x=0$ we have the identities

$$
|x|^{\top} d y^{1}(x) / d x \equiv f\left(x, y^{1}(x)\right), \quad|x|^{\top} d y^{2}(x) / d x \equiv f\left(x, y^{2}(x)\right)
$$

from which we obtain

$$
|x|^{\Gamma} \frac{d}{d x}\left(y^{1}(x)-y^{2}(x)\right) \equiv f\left(x, y^{1}(x)\right)-f\left(x, y^{2}(x)\right) .
$$

Let $z(x)=y^{1}(x)-y^{2}(x)$. We see that

$$
z(x) \rightarrow O_{n} \quad \text { as } \quad x \nearrow 0 \text {. }
$$

Since $y^{1}(x), y^{2}(x)$ are not identical in a certain neighbourhood of $x=0$, there exists a point $x_{1}$ belonging to that neighbourhood such that $\left|z\left(x_{1}\right)\right|^{2}>0$, but from the uniqueness of (3.1) $(r \geqslant 2)$ we obtain

$$
|z(x)|^{2}>0 \text { for } x \in\left\langle x_{1}, 0\right) \text {. }
$$

With the help of the mean-value theorem and by assumption 4) and (8.3) we obtain the relation

$$
f\left(x, y^{1}(x)\right)-f\left(x, y^{2}(x)\right)=(A+\varepsilon(x)) z(x),
$$

where $\delta(x)=\left[\varepsilon_{i j}(x)\right], \varepsilon_{i j}(x) \rightarrow 0$ as $x \nearrow 0, i, j=1,2, \ldots, n$. Therefore the identity (8.2) takes the form

$$
|x|^{r} d z(x) / d x \equiv(A+\varepsilon(x)) z(x) .
$$

It means that the function $z(x)$ possessing property (8.3) is the solution of the following system of differential equations:

$$
|x|^{\top} d z / d x=(A+\varepsilon(x)) z .
$$

Consider system (8.5) in the domain $\left\{(x, z): x \in(b, 0), z \in R^{n}\right\}, b \leqslant x_{1}$.

With the aid of transformation $\mathcal{G}: u=T z,|T| \neq 0,(8.5)$ is transformed into

$$
|x|^{r} d u \mid d x=G u+\bar{\varepsilon}(x) u,
$$

where $\bar{\varepsilon}(x)=\left[\bar{\varepsilon}_{i j}(x)\right], \bar{\varepsilon}_{i j}(x) \rightarrow 0$ as $x \nearrow 0$. 
Let $u(x)=T z(x)$. From (8.4) we obtain

$$
|u(x)|^{2}>0 \text { for } \quad x \in\left\langle x_{1}, 0\right) .
$$

From the fact that $u(x)$ satisfies (8.6) by virtue of inequalities (2.11), (2.12) appearing in Remark 5, we obtain

$$
|x|^{r} \frac{d}{d x}\left(|u(x)|^{2}\right) \geqslant \bar{\beta}|u(x)|^{2}-|\bar{e}(x)||u(x)|^{2},
$$

where $\bar{e}(x) \rightarrow 0$ as $x \nearrow 0, \bar{\beta}>0$.

There exists a number $x_{2}$ such that $|e(x)| \leqslant \bar{\beta} / 2$ for $x_{1} \leqslant x_{2} \leqslant x<0$. By the inequality (8.8) we have $|x|^{r} \frac{d}{d x}\left(|u(x)|^{2}\right) \geqslant(\bar{\beta} / 2)|u(x)|^{2}$ in $\left\langle x_{2}, 0\right)$.

Let us consider the ordinary differential equation

$$
d k / d x=|x|^{-r}(\bar{\beta} / 2) k \text {. }
$$

The function

$$
k(x)=k_{0} \exp \left[(\bar{\beta} / 2) \int_{x_{2}}^{x}|s|^{-r} d s\right], \quad x \in\left\langle x_{2}, 0\right)
$$

is an integral of (8.9) passing through the point $\left(x_{2}, k_{0}\right)$, where $k_{0}=\left|u\left(x_{2}\right)\right|^{2}>0$.

Hence we obtain

$$
k(x)>N \exp \left[(\bar{\beta} /(2 r-2))(-x)^{-r+1}\right], \quad x \epsilon\left\langle x_{2}, 0\right),
$$

where $N$ is a positive constant, $r \geqslant 2, \bar{\beta}>0$.

Hence we obtain

$$
k(x) \rightarrow+\infty \text { as } \quad x \nearrow 0 .
$$

From the theorem of ordinary differential inequalities [8] we obtain

$$
|u(x)|^{2} \geqslant k(x) \quad \text { for } \quad x \in\left\langle x_{2}, 0\right) .
$$

From (8.10) we have $|u(x)|^{2} \rightarrow+\infty$ as $x \nearrow 0$. From this property a contradiction can easily be obtained.

\$ 9. Proof of Theorem 6. Consider system (5.13) and denote its right-hand sides by $h^{1}(x, v, w), h^{2}(x, v, w)$. It can be shown that the system

$$
d v / d x=|x|^{-r} h^{1}(x, v, w), \quad d w / d x=|x|^{-\tau} h^{2}(x, v, w)
$$

satisfies on certain neighbourhood $N$ of the origin, for $x<0$ or $x>0$, the assumptions of a theorem of $A$. Plis [5]. Therefore the set of points lying on the integrals of (5.13) remaining in $N$ is a graph of a single-valued function defined on a certain $(n-l+1)$-dimensional set; moreover, this single-valued function satisfies the Lipschitz condition with respect to all variables. 
It can be shown as in $\S 5$ that for $(\widetilde{x}, \widetilde{w})$ from a sufficiently small neighbourhood of the origin, $\widetilde{x}<0$, there exists an integral $v(x), w(x)$ remaining in $N$ for $\tilde{x} \leqslant x<0$, having asymptotic development (5.9) and such that $w(\tilde{x})=\widetilde{w}$. Therefore by the previous remark every integral of the kind considered has the same asymptotic development.

Returning to the coordinates $x, y$ we obtain a Lipschitzian manifold consisting of points lying on the integrals of system (3.1) $(r \geqslant 2)$, remaining is a certain neighbourhood of the origin.

\section{References}

[1] R. Bellman, Stability Theory of Differential Equations, New York, Toronto, London 1953.

[2] N. G. Bruijn, Asymptotic Methods in Analysis, Amsterdam 1858.

[3] E. A. Coddington, N. Levinson, Theory of Ordinary Differential Equations, New York, Toronto, London 1855. 1945.

[4] E. Kamke, Differentialgleichungen Lösungsmethoden und Lösungen, Leipzig

[5] A. Plis, Sets filled by asymptotic integrals of ordinary differential equations, Bull. Acad. Polon. Sci., Cl III, 4 (1956), pp. 749-752.

[6] J. Szarski, Differential inequalities, Warszawa 1965.

[7] T. Ważewski, Sur un principe topologique de l'examen de l'allure asymptotique des intégrales des équations différentielles ordinaires, Ann. Soc. Polon. Math. 20 (1947), pp. 279-313.

[8] H. Whitney, Analytic extensions of differentiable functions defined in closed sets, Trans. Amer. Math. Soc. 36 (1934), p. 65. 